\title{
Improved treatment response to dornase alfa in cystic fibrosis patients using controlled inhalation
}

\author{
E.M. Bakker*, S. Volpi ${ }^{\#}$, E. Salonini ${ }^{\#}$, E.C. van der Wiel-Kooij*, \\ C.J.J.C.M. Sintnicolaas ", W.C.J. Hop ${ }^{+}$, B.M. Assael ${ }^{\#}$, \\ P.J.F.M. Merkus and H.A.W.M. Tiddens*
}

ABSTRACT: Better treatment of obstructed small airways is needed in cystic fibrosis. This study investigated whether efficient deposition of dornase alfa in the small airways improves small airway obstruction.

In a multicentre, double-blind, randomised controlled clinical trial, cystic fibrosis patients on maintenance treatment with $\mathbf{2 . 5} \mathbf{~ m L}$ dornase alfa once daily were switched to a smart nebuliser and randomised to small airway deposition $(n=24)$ or large airway deposition $(n=25)$ for 4 weeks. The primary outcome parameter was forced expiratory flow at $75 \%$ of forced vital capacity (FEF75\%).

FEF75\% increased significantly by 0.7 SD (5.2\% predicted) in the large airways group and $1.2 \mathrm{SD}$ (8.8\% pred) in the small airways group. Intention-to-treat analysis did not show a significant difference in treatment effect between groups. Per-protocol analysis, excluding patients not completing the trial or with adherence $<70 \%$, showed a trend $(p=0.06)$ in FEF75\% Z-score and a significant difference $(p=0.04)$ between groups in absolute FEF75\% $\left(L \cdot s^{-1}\right)$ favouring small airway deposition.

Improved delivery of dornase alfa using a smart nebuliser that aids patients in correct inhalation technique resulted in significant improvement of $\mathrm{FEF}_{75 \%}$ in children with stable cystic fibrosis. Adherent children showed a larger treatment response for small airway deposition.

KEYWORDS: Aerosol deposition, inhaled drug delivery, nebuliser, paediatric, small airways

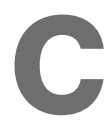
ystic fibrosis (CF) lung disease is characterised by chronic airway infection and inflammation that start early in life and cause progressive structural lung damage, which eventually leads to respiratory failure and early death [1-3]. Small airways are involved early in the disease process [4], as indicated by trapped air on CT scans $[5,6]$ and by lung function tests $[7,8]$ of young children.

Sputum of CF patients is rich in leukocyte-derived DNA that contributes to its abnormal viscoelasticity, which in turn leads to small airway obstruction [9]. A major challenge in the treatment of $\mathrm{CF}$ is, therefore, to mobilise as much sputum as possible from the airways. Airway clearance techniques and nebulisation of mucolytic drugs play an important role in mobilising sputum.

Recombinant human DNase (dornase alfa) is a mucolytic drug that has been extensively investigated in $\mathrm{CF}$ and is part of current standard treatment. Dornase alfa cleaves extracellular DNA through hydrolysis and reduces the viscoelasticity of CF sputum in vitro [10]. Dornase alfa has been shown to improve lung function and reduce the number of pulmonary exacerbations in $\mathrm{CF}$ patients [11-13]. In a study in children with normal forced vital capacity (FVC), a treatment benefit of $7.9 \%$ predicted was observed for the forced expiratory flow between $25 \%$ and $75 \%$ of FVC (FEF25-75\%), compared with a benefit of only 3.2\% pred in the primary outcome parameter, forced expiratory volume in $1 \mathrm{~s}$ (FEV1) [13]. FEF25-75\% and forced expiratory flow at 75\% of FVC (FEF75\%) are considered sensitive to changes in the small airways [14].

Because most CF patients show lung function evidence of persistent small airway obstruction $[13,15]$ and patients with advanced lung disease have extensive destruction of the small airways $[16,17]$, more efficient treatment of the small airways is still needed [18]. This could be achieved
AFFILIATIONS

*Dept of Paediatric Pulmonology and Allergology, Erasmus MC - Sophia Children's Hospital,

+Dept of Biostatistics, Erasmus MC, Rotterdam,

-Dept of Paediatric Pulmonology, Radboud University Nijmegen Medical Center, Nijmegen, The Netherlands.

"Cystic Fibrosis Center, Verona, Italy.

CORRESPONDENCE H.A.W.M. Tiddens

Erasmus MC - Sophia Children's

Hospital

Room Sp-3570

PO Box 2060

3000 CB Rotterdam

The Netherlands

E-mail: h.tiddens@erasmusmc.nl

Received:

Jan 132011

Accepted after revision:

May 142011

First published online:

July 072011 
through improved delivery of dornase alfa to the small airways. Currently, dornase alfa is routinely administered with relatively inefficient nebulisers. These nebulisers deposit most of the inhaled drug in large airways and relatively little of the drug in the small airways $[19,20]$. This is primarily due to two nebuliser-related factors: particle size of the inhaled aerosol and breathing pattern during inhalation. Standard jet nebulisers produce an aerosol with a wide range of particle sizes and a relatively large mean particle size. Large aerosol particles have a high probability to get deposited in the central airways. Furthermore, most patients perform tidal breathing while using a standard nebuliser. This leads to preferential deposition of particles in the large airways. In contrast, a slow and deep inhalation increases the probability of particle deposition in the small airways.

Recently, smart nebulisers have become available that coach patients in proper inhalation technique, are more efficient in drug delivery and can target more of the inhaled dose to the small airways [21-23]. However, it is unknown whether preferential delivery of dornase alfa to small airways using a state-of-the-art nebuliser actually improves small airway patency in children with CF. To answer this question, we conducted a randomised controlled clinical trial comparing large and small airway deposition of dornase alfa in stable CF patients using a state-of-the-art nebuliser.

\section{METHODS}

We conducted a multicentre, double-blind, randomised controlled clinical trial in stable paediatric CF patients who were on maintenance treatment with $2.5 \mathrm{mg}$ dornase alfa once daily, to compare the efficacy of small airway versus large airway deposition of dornase alfa using a breath-controlled nebuliser.

\section{Patient recruitment and randomisation}

Study participants were recruited from the outpatient clinics of three participating hospitals: Erasmus MC - Sophia Children's Hospital (Rotterdam, the Netherlands), Radboud University Nijmegen Medical Centre (Nijmegen, the Netherlands) and the Cystic Fibrosis Center (Verona, Italy).

CF patients were eligible to participate in the study if they were aged 6-18 yrs, in stable condition, on maintenance treatment with $2.5 \mathrm{mg}$ dornase alfa once daily and had small airway obstruction present on spirometry (defined as FEF75\% $<70 \%$ pred). A full checklist of inclusion, exclusion and study stop criteria used in the study is available in the online supplementary material.

Patients were randomly assigned to the small airway deposition group or the large airway deposition group, according to the randomisation schedule prepared by the study statistician. The hospital pharmacy was responsible for the administration of randomisation data and guaranteed 24-h availability of randomisation information.

This trial was registered in the Dutch trial register (www. trialregister.nl) and in the International Standard Randomised Controlled Trial Number Register (number 64225851). The local ethics committee approved the study. Parental written informed consent and children's assent were obtained prior to the first study measurement.

\section{Study treatment}

Study treatment consisted of targeted peripheral or central deposition of $2.5 \mathrm{mg}(2.5 \mathrm{~mL})$ dornase alfa. Central deposition simulated conventional nebulisers and deposited most of the drug in the large airways (large airways group), while peripheral deposition optimised deposition in the small airways (small airways group) [21, 24-26]. Dornase alfa was inhaled once daily using the Akita ${ }^{2}$ APIXNEB device (Activaero Technologies, Gemuenden, Germany). This device consists of a nebuliser handset (APIXNEB) that uses vibrating mesh technology (Touchspray; PARI GmbH, Starnberg, Germany) and an electronic unit $\left(\mathrm{Akita}^{2}\right)$. In this publication, we will refer to this device as Akita $^{2}$. The Akita ${ }^{2}$ deposits $\sim 70 \%$ of the loaded dose in the lung [26], compared with 10-20\% for conventional jet nebuliser systems [27-30]. Therefore, total lung dose of dornase alfa during this study was estimated to be 3-5 times higher than with standard jet nebulisers. The Akita ${ }^{2}$ can be programmed to preferentially target specific regions of the lung [21, 24-26]. The Akita ${ }^{2}$ directs the flow and depth of each inhalation, coaches the patient on correct inhalation technique and controls the fraction of the inspiration time during which aerosol is generated. These settings are optimised for each patient based on individual inspiratory capacity using a microchip-containing smartcard inserted in the device.

In order to generate the two different lung deposition patterns, we adjusted three characteristics of the nebuliser treatment: particle size, breathing pattern and timing of aerosol bolus (fig. 1).

The investigator carefully instructed each patient on the use of the $\mathrm{Akita}^{2}$ at the start of the study. Patients in both treatment arms received instructions and feedback from the display of the $\mathrm{Akita}^{2}$ during each nebulisation, to ensure that the correct

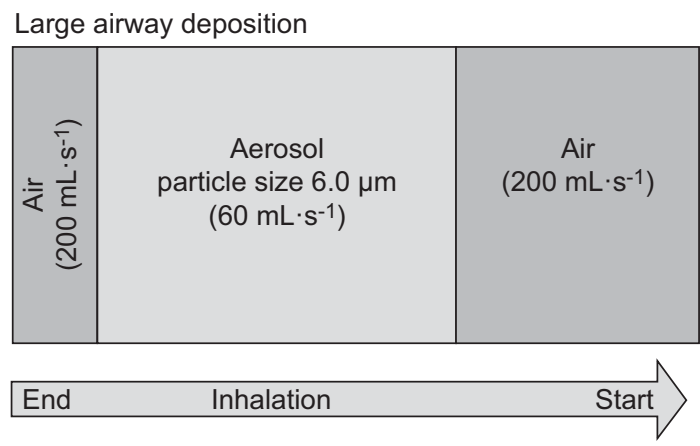

Small airway deposition

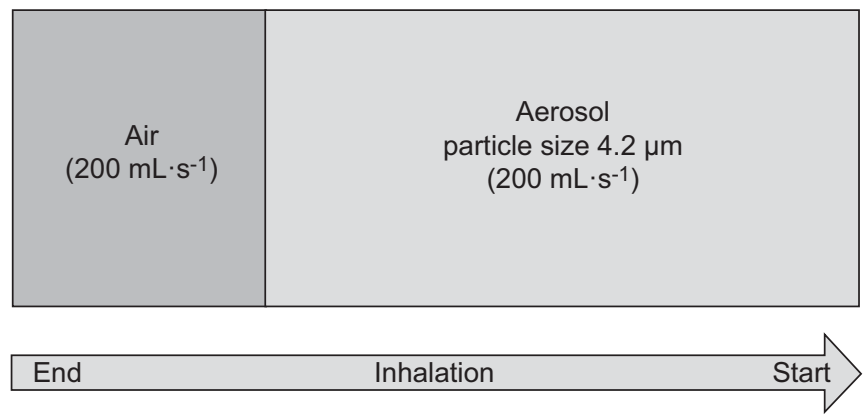

FIGURE 1. Breathing patterns on smartcard. 
breathing pattern was performed. Additional details on feedback to the patient and inhalation settings of the device, as well as technical details, can be found in the online supplementary material.

\section{Study end-points}

The primary end-point of this study was change in FEF75\% (Z-score) after 4 weeks of targeted treatment. Secondary endpoints were change in FEF75\% $\left(\mathrm{L} \cdot \mathrm{s}^{-1}\right)$, change in $\mathrm{FEV} 1, \mathrm{FVC}$, FEF25-75\% (Z-score and absolute values), lung clearance index (LCI) and diary symptom scores.

Study visits were scheduled at $\mathrm{t}=0,2$ and 4 weeks. At each study visit, spirometry tests were performed. Spirometry was measured on a Masterscreen electronic spirometer (Jaeger/Viasys, Würzburg, Germany) according to European Respiratory Society/American Thoracic Society guidelines. LCI measured by a multiple breath washout test (Exhalyzer ${ }_{\circledR} \mathrm{D}$ Ecomedics, Duernten, Switzerland) was performed at each visit only in the Sophia Children's Hospital because this test was not available in the other two centres.

Adverse events during the study were recorded in an updated version of a diary used in previous studies (table E2 of the online supplementary material) [31,32]. Patients were asked to daily record any unusual symptoms and a symptom score comprising items on cough, mucus and shortness of breath. The possible total symptom score for 1 day ranged from 0 to 18 points.

\section{Adherence}

The Akita ${ }^{2}$ monitors adherence by recording on the smartcard the date, time and number of breaths of each nebulisation treatment. For each patient, we calculated daily dose adherence, which expresses the percentage of days a patient adhered correctly to the prescribed once daily nebulisation of dornase alfa.

\section{Estimate of sample size}

We aimed to detect a $10 \%$ difference in FEF75\% at the 5\% significance level for a two-sided test with $80 \%$ power. Based on previous data, this would require 44 patients in total (22 patients in each group) [33]. To anticipate possible dropouts, we aimed to include 25 patients per study arm.

\section{Statistical analysis}

Data were analysed on an intention-to-treat basis. Analyses of between-group comparisons regarding change in lung function variables (FVC, FEV1, FEF75\%, FEF25-75\% and LCI) were performed using repeated measurements ANOVA with adjustment for baseline values, centre and use of tobramycin solution for inhalation. Changes are reported as mean \pm SEM. Analyses were performed with SPSS software (version 15.0; IBM, Chicago, IL, USA). For all analyses, two-tailed p-values were considered to indicate statistical significance when $\mathrm{p}<0.05$. A per-protocol analysis was conducted in which patients who violated the study protocol were excluded. Protocol violations were defined as patients who did not complete the trial, had adherence $<70 \%$ or for whom adherence data could not be retrieved (fig. 2).

Patients who improved by $\geqslant 10 \%$ pred for FEF75\% were defined as excellent responders. We calculated whether there

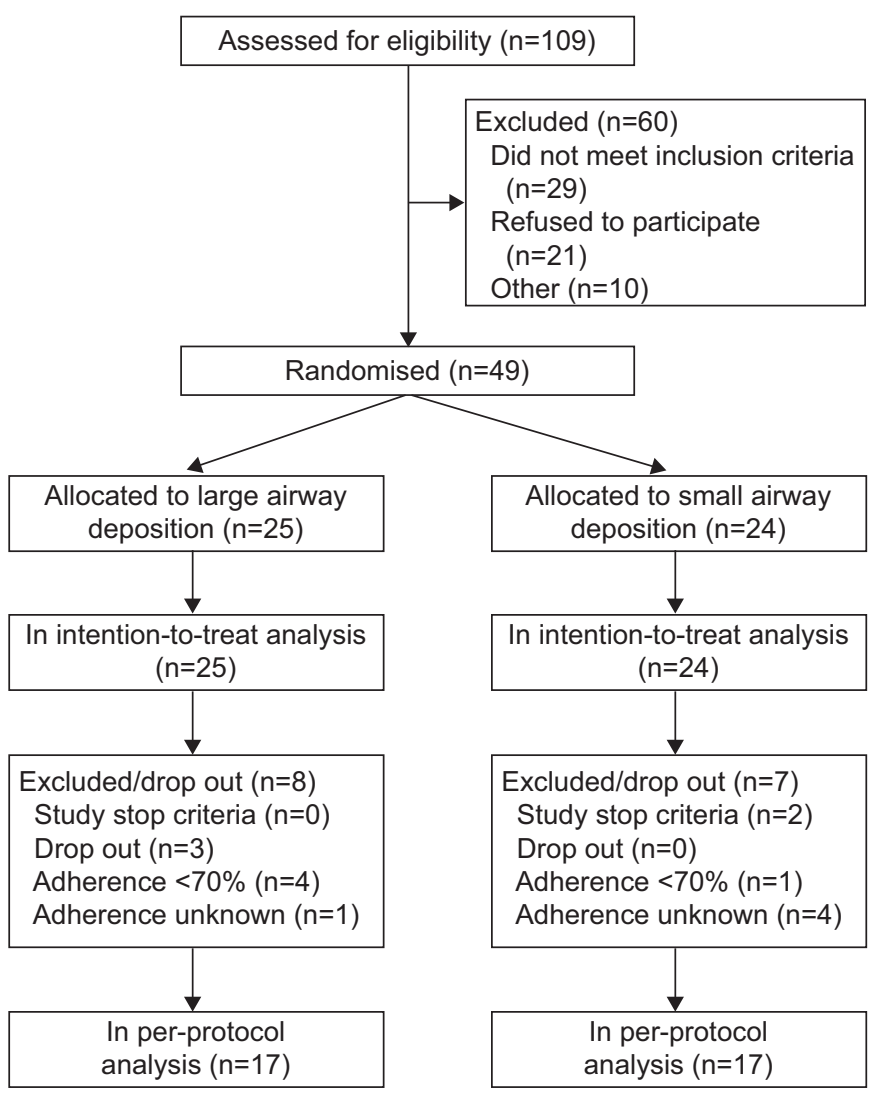

FIGURE 2. Enrolment, random assignment, follow-up and analysis.

was a significant difference between the groups in number of excellent responders using a Chi-squared test with continuity correction.

Spirometry variables were expressed as Z-scores and \% pred using the reference values published by STANOJEVIC et al. [34] for FVC, FEV1 and FEF25-75\%. Since these reference equations do not include data for FEF75\%, we used the reference values published by ZAPLETAL et al. [35] for FEF25-75\%. Changes in absolute values of spirometry variables $\left(\mathrm{L}\right.$ and $\mathrm{L} \cdot \mathrm{s}^{-1}$ ) were expressed as percentage of baseline values.

For each patient, mean daily symptom scores per week were computed. When $<4$ out of 7 days of a week were recorded, scores for that week were regarded as not evaluable. Differences in number of adverse events and changes in diary scores were analysed using a Mann-Whitney test and Chisquare test.

\section{RESULTS}

49 patients were included in the study, 44 of whom completed the trial (22 patients in each group). All patients were enrolled between September 2007 and March 2010. Baseline characteristics of patients in the two treatment groups were similar (table 1). Mean treatment time for the high lung dose of dornase alfa that was delivered during this study was $11 \mathrm{~min} 51 \mathrm{~s}$ for the large airways group and $9 \mathrm{~min} 27 \mathrm{~s}$ for the small airways group. Difference in treatment time between the groups was not significant $(\mathrm{p}=0.13)$. 


\begin{tabular}{|c|c|c|c|}
\hline \multirow{2}{*}{$\begin{array}{l}\text { TABLE } 1 \\
\text { Variables }\end{array}$} & \multicolumn{3}{|c|}{$\begin{array}{l}\text { Baseline characteristics of patients in each } \\
\text { treatment group }\end{array}$} \\
\hline & & Large airways & Small airways \\
\hline \multicolumn{2}{|l|}{ Subjects } & 25 & 24 \\
\hline \multicolumn{2}{|c|}{ Sex males/females } & $14 / 11$ & $12 / 12$ \\
\hline \multicolumn{2}{|l|}{ Age yrs } & $13.3 \pm 3.4$ & $12.7 \pm 3.5$ \\
\hline \multicolumn{2}{|l|}{ Height $\mathrm{cm}$} & $153.5 \pm 16.5$ & $153.4 \pm 18.1$ \\
\hline \multicolumn{2}{|l|}{ BMI $\mathbf{k g} \cdot \mathrm{m}^{-2}$} & $18.7 \pm 2.7$ & $18.2 \pm 2.2$ \\
\hline \multicolumn{2}{|c|}{ Pancreatic insufficiency yes/no } & $22 / 3$ & $20 / 4$ \\
\hline \multicolumn{2}{|c|}{ Chronic $P$. aeruginosa yes/no } & $10 / 15$ & $9 / 15$ \\
\hline \multicolumn{2}{|c|}{ Maintenance inhaled tobramycin yes/no } & $9 / 16$ & $8 / 16$ \\
\hline \multicolumn{2}{|c|}{ CF mutation homozygous delF508/other } & $15 / 10$ & $10 / 14$ \\
\hline \multicolumn{2}{|l|}{ FVC Z-score } & $-0.5 \pm 1.2$ & $-0.7 \pm 1.5$ \\
\hline \multicolumn{2}{|l|}{ FVC $\%$ pred } & $94.6 \pm 13.1$ & $91.7 \pm 17.1$ \\
\hline \multicolumn{2}{|c|}{ FEV1 Z-score } & $-1.6 \pm 1.1$ & $-1.7 \pm 1.3$ \\
\hline \multicolumn{2}{|c|}{ FEV $1 \%$ pred } & $81.8 \pm 13.1$ & $80.4 \pm 15.6$ \\
\hline \multicolumn{2}{|c|}{ FEF25-75\% Z-score } & $-2.2 \pm 1.2$ & $-2.3 \pm 1.2$ \\
\hline \multicolumn{2}{|c|}{ FEF $25-75 \% \%$ pred } & $55.5 \pm 19.6$ & $54.7 \pm 19.9$ \\
\hline \multicolumn{2}{|c|}{ FEF75\% Z-score } & $-5.2 \pm 2.7$ & $-5.5 \pm 2.7$ \\
\hline \multicolumn{2}{|c|}{ FEF75\% \% pred } & $39.7 \pm 17.4$ & $37.2 \pm 16.0$ \\
\hline \multicolumn{2}{|c|}{$\mathrm{LCl}$ absolute values } & $8.7 \pm 1.6^{\#}$ & $9.9 \pm 1.8^{\bullet}$ \\
\hline
\end{tabular}

Data are reported as $\mathrm{n}$ or mean $\pm \mathrm{SD}$. BMI: body mass index; $P$. aeruginosa: Pseudomonas aeruginosa; CF: cystic fibrosis; FVC: forced vital capacity; \% pred: \% predicted; FEV1: forced expiratory volume in $1 \mathrm{~s}$; FEF25-75\%: forced expiratory flow between $25 \%$ and $75 \%$ of FVC; FEF $75 \%$ : forced expiratory flow at $75 \%$ of FVC; LCl: lung clearance index. ${ }^{*}: \mathrm{n}=15 ; ": n=14$.

\section{Lung function (intention-to-treat analysis)}

FEF75\% improved substantially and significantly in both groups after 4 weeks of treatment (table 2 and fig. 3a): by $0.7 \mathrm{SD}(\mathrm{p}=0.03)$ in the large airways group and by $1.2 \mathrm{SD}$ $(p=0.001)$ in the small airways group. This corresponds to an improvement of $5.2 \%$ and $8.8 \%$ pred, respectively. The difference in mean FEF75\% between both groups was $0.4 \mathrm{SD}$ (95\% CI $-0.4-1.2 \mathrm{SD} ; \mathrm{p}=0.31$ ) for small airways versus large airways deposition.

A similar effect was observed for FEF25-75\% (table 2). FVC and FEV1 did not change significantly in both groups, and the difference in FEV1 or FVC between the groups after 4 weeks of treatment was not significant.

FEF75\% improved by $\geqslant 10 \%$ pred in four $(18 \%)$ out of 22 patients in the large airways group and in $10(45 \%)$ out of 22 patients in the small airways group. The difference in number of excellent responders between the groups was not significant $(\mathrm{p}=0.10)$.

LCI was measured in 29 patients: 15 in the large airways and 14 in the small airways group. LCI did not change significantly in either group over 4 weeks, nor was there a significant difference between the groups in the change in LCI (table 2).

\section{Symptom scores}

Mean symptom score per day in week 1 was $1.8 \pm 1.9$ points for the large airways group and $2.9 \pm 2.3$ points in the small airways group. Symptom scores were slightly lower in the first week of the study in the large than the small airways group $(p=0.05)$. Mean symptom scores during the rest of the study did not significantly differ between treatment groups and there were no significant changes within either group (table E3 of the online supplementary material).

\section{FEF75\% (per-protocol analysis)}

15 out of the 49 patients were excluded from the per-protocol analysis: five patients did not complete the trial, five patients had a daily dose adherence $<70 \%$ and, for five patients, data on adherence could not be retrieved (fig. 2). As a result, 17 patients in each group were analysed for the primary endpoint (FEF75\%).

TABLE 2 Lung function after 4 weeks of study treatment

\begin{tabular}{|c|c|c|c|c|c|}
\hline \multirow[t]{2}{*}{ Variables } & \multicolumn{2}{|c|}{ Large airways $\mathrm{ITT}^{\#}$} & \multicolumn{2}{|c|}{ Small airways ITT" } & \multirow{2}{*}{$\begin{array}{c}\text { Mean difference ITT } \\
\text { Z-score }(95 \% \mathrm{Cl})\end{array}$} \\
\hline & Z-score & $\mathrm{L} \cdot \mathrm{s}^{-1} \% \mathrm{BL}$ & Z-score & $\mathrm{L} \cdot \mathrm{s}^{-1} \% \mathrm{BL}$ & \\
\hline FEF75\% & $0.7 \pm 0.3^{\star}$ & $24.1 \pm 10.3^{\star}$ & $1.2 \pm 0.3^{*}$ & $35.3 \pm 10.1^{*}$ & $0.4(-0.4-1.2)$ \\
\hline FVC & $0.0 \pm 0.1$ & $1.4 \pm 1.5$ & $0.1 \pm 0.1$ & $2.4 \pm 1.5$ & $0.0(-0.2-0.3)$ \\
\hline FEV 1 & $0.2 \pm 0.1$ & $3.6 \pm 2.1$ & $0.1 \pm 0.1$ & $3.5 \pm 2.1$ & $-0.1(-0.4-0.3)$ \\
\hline \multirow{2}{*}{$\mathrm{LCl}$} & \multicolumn{2}{|c|}{ Large airways $\mathrm{PP} f$} & \multicolumn{2}{|c|}{ Small airways $\mathrm{PP}^{f}$} & Mean difference PP \\
\hline & Z-score & $\mathrm{L} \cdot \mathrm{s}^{-1} \% \mathrm{BL}$ & Z-score & $\mathrm{L} \cdot \mathrm{s}^{-1} \% \mathrm{BL}$ & Z-score $(95 \% \mathrm{Cl})$ \\
\hline FEF $75 \%$ & $0.7 \pm 0.3^{*}$ & $17.3 \pm 7.5^{\star, \# \#}$ & $1.4 \pm 0.3^{*}$ & $37.5 \pm 7.5^{\star, \# \#}$ & $0.7(-0.1-1.5)$ \\
\hline
\end{tabular}

Data are presented as mean \pm SEM change from baseline (ANOVA estimates), unless otherwise stated. Difference is shown for small airways minus large airways group (Z-score and 95\% CI). ITT: intention-to-treat analysis; \% BL: \% change from baseline; FVC: forced vital capacity; FEF75\%: forced expiratory flow at $75 \%$ of FVC; FEV1: forced expiratory volume in $1 \mathrm{~s}$; FEF25-75\%: forced expiratory flow between $25 \%$ and $75 \%$ of FVC; LCl: lung clearance index; PP: per-protocol analysis. PP analysis was performed only for the primary end-point, FEF75\%. ${ }^{\#}: n=25 ;{ }^{\circ}: n=24 ;^{+}: n=15 ;{ }^{5}: n=14 ;: n=17 . *: p \leqslant 0.05$ for change from baseline within treatment group; $\# \#: p \leqslant 0.05$ for difference between treatment groups. 

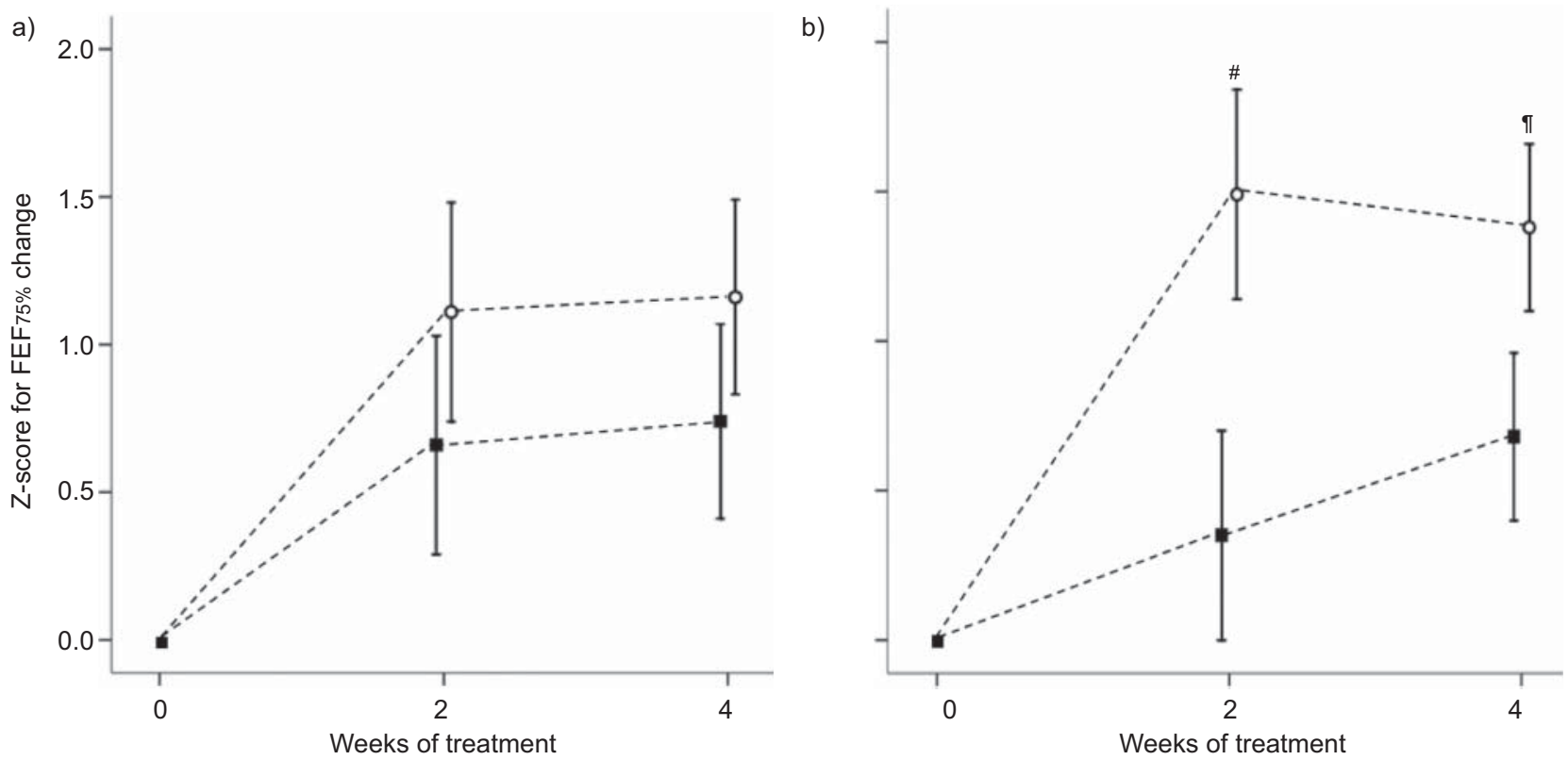

FIGURE 3. Mean changes from baseline for forced expiratory flow at $75 \%$ of forced vital capacity (FEF75\%) (Z-score) after 2 and 4 weeks of treatment. a) Intention-totreat analysis; b) per-protocol analysis. Data are presented as mean \pm SEM (ANOVA estimates). Closed squares represent large airway deposition, open circles represent small airway deposition. ${ }^{\#}: p=0.03 ;{ }^{\imath}: p=0.06$, for small versus large airway deposition.

FEF75\% improved significantly in both groups after 4 weeks of treatment: by 0.7 SD $(95 \%$ CI $0.1-1.2 \mathrm{SD} ; \mathrm{p}=0.02)$ in the large airways group, and by 1.4 SD $(95 \%$ CI $0.8-1.9$ SD; $\mathrm{p}<0.001)$ in the small airways group (table 2). These increases in Z-scores correspond with improvements of $5.6 \%$ and $10.7 \%$ pred, respectively. Analysis of change in absolute values of FEF75\% $\left(\mathrm{L} \cdot \mathrm{s}^{-1}\right.$ and expressed as percentage of baseline) demonstrated that FEF75\% improved by $17.3 \%(95 \%$ CI $2.1-32.6 \%$; $<<0.001)$ in the large airways group and by $37.5 \%$ (95\% CI $22.2-52.8 \%$; $\mathrm{p}<0.001$ ) in the small airways group.

After 2 weeks of treatment, the difference in FEF75\% between the groups was $1.2 \mathrm{SD}(95 \% \mathrm{CI} 0.1-2.2 \mathrm{SD} ; \mathrm{p}=0.03)$ in favour of the small airways group. After 4 weeks, the difference was 0.7 SD (95\% CI $-0.1-1.5 \mathrm{SD} ; \mathrm{p}=0.06)$ in favour of the small airways group (fig. 3b). Expressed as absolute values, the difference in FEF75\% in favour of small airways deposition was $32.4 \%(95 \%$ CI $3.4-61.4 \% ; \mathrm{p}=0.03)$ after 2 weeks and $21.3 \%$ (95\% CI 1.4-41.2\%; $\mathrm{p}=0.04$ ) after 4 weeks of treatment (data shown in figure E1 of the online supplementary material).

\section{Adverse events}

A total of 31 adverse events were reported during the study, 18 in the large airways group and 13 in the small airways group. There was no statistically significant difference in number of adverse events between the groups $(p=0.90)$.

In general, adverse events were either self-limiting symptoms (e.g. runny nose and headache) or symptoms consistent with CF (e.g. respiratory infections and bowel problems). Three adverse events were considered to be possibly related to the study intervention: three patients in the large airways group experienced hoarseness for 1 or 2 days during the first 2 weeks of the study. Temporary hoarseness is a known side-effect of dornase alfa inhalation. In these patients the symptoms resolved spontaneously. No serious adverse events were reported in either group.

\section{DISCUSSION}

To our knowledge, this is the first randomised double-blind controlled trial comparing large airway versus small airway deposition of inhaled dornase alfa using a smart nebuliser system. We observed a substantial and statistically significant improvement in spirometry indicators of small airway patency in both groups. The per-protocol analysis, but not the intention-to-treat analysis, demonstrated better treatment response in the small airways group.

The most striking finding in this study is that both treatment groups showed considerable improvement in FEF75\% and FEF25-75\%, indicating improved patency of the small airways, within 4 weeks of switching from a conventional nebuliser to a smart nebuliser for their daily dornase alfa inhalation. Mean improvement in FEF25-75\% after 4 weeks of treatment for all patients, independent of deposition group, was 5.9\% pred. This is remarkable, because all patients were on maintenance treatment with the same nominal dose of dornase alfa prior to the study. It is noteworthy that this improvement is relatively large compared with the $8.4 \%$ improvement in the PEIT study [13], which included dornase alfa-naïve patients who were randomised to $2.5 \mathrm{mg}$ dornase alfa or placebo once daily with a conventional jet nebuliser. Our study included only patients who were already on maintenance dornase alfa treatment and we simply changed the nebuliser. Hence, the present study suggests that the efficacy of the same medication can be improved by another $70 \%$ simply by optimising adherence and inhalation technique.

There are a few explanations for this improvement of small airway function. One likely explanation is that adherence to 
dornase alfa treatment was higher during the study than before it. Overall adherence to once daily dornase alfa nebulisation was $85 \%$ during the study and thus probably above daily routine. It is well-known that adherence to chronically inhaled medication is in the order of $40-60 \%$ in CF patients and is higher during clinical trials [36-38]. However, we feel that the improvement in FEF75\% is too large to be caused by improved adherence only.

A second likely explanation for the improvement in FEF75\% in both groups is the controlled inhalation technique of the smart nebuliser. The Akita $^{2}$ device directs inspiratory flow and inhaled volume during each breath and coaches patients to perform the correct breathing manoeuvre. Consequently, the use of this device increases total lung dose of dornase alfa, improves small airways deposition and reduces loss of aerosol into the environment [21, 24-26].

A third explanation is the higher lung dose delivered during study treatment. Before enrolment in this study, $84 \%$ of the patients used a conventional jet nebuliser for their maintenance treatment (table E4 of the online supplementary material). These nebulisers have poor efficiency, delivering only $10-20 \%$ of the loaded dose to the lungs [27-30]. Up to $50 \%$ of the drug is retained in the nebuliser at the end of nebulisation, and $20 \%$ of the nominal dose is lost to the environment [28]. The smart nebuliser used in our study delivers up to $70 \%$ of the nominal dose to the lungs $[24,26]$.

At the time of the registration studies of dornase alfa several dose finding studies were performed. Daily doses varying between 2.5 and $20 \mathrm{mg}$ were given to patients in phase II and III trials without observing a dose-dependent response in FEV1 or FVC [11, 39]. All doses were safe. Unfortunately, these studies did not report the effect of the various treatment regimens on $\mathrm{FEF} 75 \%$ or $\mathrm{FEF} 25-75 \%$.

The other important new finding of this study is that increased delivery of dornase alfa to small airways is possible, as demonstrated by greater FEF75\% and FEF25-75\% responses in both treatment groups, and that doing so seems to be safe. Small airway deposition was accomplished by reducing aerosol particle size and nebulising drug early during deeper inhalation, but even the large airways group benefitted from an increased peripheral deposition. The intention-to-treat analysis did not show a difference between the two deposition groups. This might be explained by the fact that the use of a breath-actuated and efficient nebuliser in this population with relatively mild airway obstruction resulted in substantially more drug reaching the large surface area of the small airways than when using a conventional jet nebuliser, even for the central airways deposition settings. This might have resulted in reaching the plateau of the dose-response curve in both groups and thus not finding a significant difference between the two deposition groups.

However, the per-protocol analysis demonstrated greater improvement and a significant difference between the groups for FEF75\% $\left(\mathrm{L} \cdot \mathrm{s}^{-1}\right)$ in favour of the small airways group. This "best case scenario" analysis illustrates the potential of optimising every possible aspect of dornase alfa therapy, i.e. optimal delivery to the small airways and optimal adherence. The clinical relevance of our study is that it underlines that both adherence and inhalation competence are important determinants for the optimisation of the treatment effect of dornase alfa. Clearly, the use of a breath-actuated efficient nebuliser for maintenance treatment of dornase alfa should be considered "off-label treatment". However, nowadays most patients use off-label nebulisers because these are different from the ones used in the registration studies for dornase alfa almost two decades ago. We feel that a breath-actuated efficient nebuliser is a promising treatment option that should be considered, especially for those patients with progressive small airway disease or possible poor competence and/or adherence.

Two previous large, open-label studies in dornase alfa-naïve patients compared the efficacy of small versus larger particles and showed a similar trend as in this study, but were not conclusive [40, 41]. The most likely explanation for these negative findings was the use of conventional jet nebuliser systems. Standard jet nebulisers produce an aerosol with a wide range of particle sizes and a relatively large mean particle size. Large aerosol particles have a high probability for deposition in the central airways. Furthermore, most patients perform tidal breathing while using a standard nebuliser. This leads to preferential deposition of particles in the large airways. In contrast, a slow and deep inhalation increases the probability of particle deposition in the small airways. Therefore, the conditions in these two studies probably were not optimal for delivery of the drug to the small airways. An open-label, singlearm study on bolus inhalation of dornase alfa by GELLER et al. [42] showed the importance of breath control during nebulisation. In this study, CF patients were treated with a device that was breath actuated, delivered an aerosol bolus when the optimal inspiratory flow rate was achieved and generated visual cues for the subject to inhale deeply within the optimum range of inspiratory flow rates. FEV1 and FEF25-75\% significantly improved after 2 weeks of dornase alfa treatment using the study device. Our study supports the conclusions by GELLER et al. [42], that controlled and deep inhalation is important in order to achieve optimal treatment effect.

It is likely that the optimised inhalation technique used in our study could also benefit patients with other respiratory diseases, such as chronic obstructive pulmonary disease or asthma. However, this should be confirmed by clinical trials conducted in these patient groups.

In conclusion, our study shows that 4 weeks of dornase alfa nebulised using a state-of-the-art nebuliser device resulted in a significant improvement in FEF75\% and FEF25-75\% in children with stable CF who were on prior maintenance treatment with dornase alfa. In children with good adherence (per-protocol analysis), greater treatment response was observed for small airway deposition compared with large airway deposition.

CF is a fatal disease and its main cause of death is loss of lung function with respiratory failure. Every option to improve lung function and to reduce its rate of decline should be utilised in order to improve its long-term prognosis. This study suggests that better treatment response and further improvement of small airway function is indeed feasible by optimising the inhalation technique for administration of a known and effective mucolytic drug, using an efficient nebuliser and breath-control technology. 


\section{CLINICAL TRIAL}

This study is registered at www.trialregister.nl and in the International Standard Randomised Controlled Trial Number Register with identifier number 6422851 .

\section{STATEMENT OF INTEREST}

Statements of interest for E.M. Bakker, B.M. Assael and H.A.W.M. Tiddens, and for the study itself, can be found at www.erj.ersjournals. $\mathrm{com} / \mathrm{site} / \mathrm{misc} /$ statements.xhtml

\section{ACKNOWLEDGEMENTS}

We thank all patients and parents, all medical personnel at the study sites, and I. Meneghelli and M. Passiu at the CF centre in Verona, Italy for their contributions to this study, and we thank S. McKenzie (Victoria, Canada) for her critical reading of the manuscript. We thank Activaero $\mathrm{GmbH}$ (Gemuenden, Germany) for its support and contributions to this study.

\section{REFERENCES}

1 Dakin $\mathrm{CJ}$, Numa $\mathrm{AH}$, Wang $\mathrm{H}$, et al. Inflammation, infection, and pulmonary function in infants and young children with cystic fibrosis. Am J Respir Crit Care Med 2002; 165: 904-910.

2 Armstrong DS, Grimwood K, Carlin JB, et al. Lower airway inflammation in infants and young children with cystic fibrosis. Am J Respir Crit Care Med 1997; 156: 1197-1204

3 Nixon GM, Armstrong DS, Carzino R, et al. Early airway infection, inflammation, and lung function in cystic fibrosis. Arch Dis Child 2002; 87: 306-311.

4 Tiddens HA, Donaldson SH, Rosenfeld M, et al. Cystic fibrosis lung disease starts in the small airways: can we treat it more effectively? Pediatr Pulmonol 2010; 45: 107-117.

5 Sly PD, Brennan S, Gangell C, et al. Lung disease at diagnosis in infants with cystic fibrosis detected by newborn screening. Am J Respir Crit Care Med 2009; 180: 146-152.

6 Stick SM, Brennan S, Murray C, et al. Bronchiectasis in infants and preschool children diagnosed with cystic fibrosis after newborn screening. J Pediatr 2009; 155: 623-628.

7 Linnane BM, Hall GL, Nolan G, et al. Lung function in infants with cystic fibrosis diagnosed by newborn screening. Am J Respir Crit Care Med 2008; 178: 1238-1244.

8 Aurora P, Bush A, Gustafsson P, et al. Multiple-breath washout as a marker of lung disease in preschool children with cystic fibrosis. Am J Respir Crit Care Med 2005; 171: 249-256.

9 Lethem MI, James SL, Marriott C. The role of mucous glycoproteins in the rheologic properties of cystic fibrosis sputum. Am Rev Respir Dis 1990; 142: 1053-1058.

10 Shak S, Capon DJ, Hellmiss R, et al. Recombinant human DNase I reduces the viscosity of cystic fibrosis sputum. Proc Natl Acad Sci USA 1990; 87: 9188-9192.

11 Fuchs HJ, Borowitz DS, Christiansen DH, et al. Effect of aerosolized recombinant human DNase on exacerbations of respiratory symptoms and on pulmonary function in patients with cystic fibrosis. The Pulmozyme Study Group. N Engl J Med 1994; 331: 637-642.

12 Hodson ME, Shah PL. DNase trials in cystic fibrosis. Eur Respir J 1995; 8: 1786-1791.

13 Quan JM, Tiddens HA, Sy JP, et al. A two-year randomized, placebo-controlled trial of dornase alfa in young patients with cystic fibrosis with mild lung function abnormalities. J Pediatr 2001; 139: 813-820.

14 Gustafsson PM, De Jong PA, Tiddens HA, et al. Multiple-breath inert gas washout and spirometry versus structural lung disease in cystic fibrosis. Thorax 2008; 63: 129-134.
15 Kozlowska WJ, Bush A, Wade A, et al. Lung function from infancy to the preschool years after clinical diagnosis of cystic fibrosis. Am J Respir Crit Care Med 2008; 178: 42-49.

16 Hamutcu R, Rowland JM, Horn MV, et al. Clinical findings and lung pathology in children with cystic fibrosis. Am J Respir Crit Care Med 2002; 165: 1172-1175.

17 Tiddens HA, Koopman LP, Lambert RK, et al. Cartilaginous airway wall dimensions and airway resistance in cystic fibrosis lungs. Eur Respir J 2000; 15: 735-742.

18 Brownlee KG. Small airways disease in cystic fibrosis. Eur Respir Mon 2006; 35: 21-37.

19 Diot P, Palmer LB, Smaldone A, et al. RhDNase I aerosol deposition and related factors in cystic fibrosis. Am J Respir Crit Care Med 1997; 156: 1662-1668.

20 Laube BL, Jashnani R, Dalby RN, et al. Targeting aerosol deposition in patients with cystic fibrosis: effects of alterations in particle size and inspiratory flow rate. Chest 2000; 118: 1069-1076.

21 Brand $\mathrm{P}$, Meyer T, Haussermann S, et al. Optimum peripheral drug deposition in patients with cystic fibrosis. J Aerosol Med 2005; 18: 45-54.

22 Brand $\mathrm{P}$, Beckmann $\mathrm{H}$, Maas Enriquez $\mathrm{M}$, et al. Peripheral deposition of alpha1-protease inhibitor using commercial inhalation devices. Eur Respir J 2003; 22: 263-267.

23 Griese M, Ramakers J, Krasselt A, et al. Improvement of alveolar glutathione and lung function but not oxidative state in cystic fibrosis. Am J Respir Crit Care Med 2004; 169: 822-828.

24 Kohler E, Sollich V, Schuster-Wonka R, et al. Lung deposition after electronically breath-controlled inhalation and manually triggered conventional inhalation in cystic fibrosis patients. J Aerosol Med 2005; 18: 386-395.

25 Scheuch G, Müllinger B, Brand P, et al. Optimizing aerosol lung deposition in cystic fibrosis patients. In: Romano L, Manno G, Galietta LJV, eds. Proceedings of the 25th European Cystic Fibrosis Conference. Bologna, Monduzzi Editore, 2002; pp. 169-172.

26 Brand $\mathrm{P}$, Schulte $\mathrm{M}$, Wencker $\mathrm{M}$, et al. Lung deposition of inhaled $\alpha_{1}$-proteinase inhibitor in cystic fibrosis and $\alpha_{1}$-antitrypsin deficiency. Eur Respir J 2009; 34: 354-360.

27 Devadason SG, Everard ML, Linto JM, et al. Comparison of drug delivery from conventional versus "Venturi" nebulizers. Eur Respir J 1997; 10: 2479-2483.

28 Rau JL, Ari A, Restrepo RD. Performance comparison of nebulizer designs: constant-output, breath-enhanced, and dosimetric. Respir Care 2004; 49: 174-179.

29 Newman SP, Pitcairn GR, Hooper G, et al. Efficient drug delivery to the lungs from a continuously operated open-vent nebulizer and low pressure compressor system. Eur Respir J 1994; 7: 1177-1181.

30 Hardy JG, Newman SP, Knoch M. Lung deposition from four nebulizers. Respir Med 1993; 87: 461-465.

31 Pijnenburg MW, Bakker EM, Hop WC, et al. Titrating steroids on exhaled nitric oxide in children with asthma: a randomized controlled trial. Am J Respir Crit Care Med 2005; 172: 831-836.

32 Verberne AA, Frost C, Roorda RJ, et al. One year treatment with salmeterol compared with beclomethasone in children with asthma. The Dutch Paediatric Asthma Study Group. Am J Respir Crit Care Med 1997; 156: 688-695.

33 van der Giessen LJ, de Jongste JC, Gosselink R, et al. RhDNase before airway clearance therapy improves airway patency in children with CF. Pediatr Pulmonol 2007; 42: 624-630.

34 Stanojevic S, Wade A, Stocks J, et al. Reference ranges for spirometry across all ages: a new approach. Am J Respir Crit Care Med 2008; 177: 253-260.

35 Zapletal A, Paul T, Samanek M. Die Bedeutung heutiger Methoden der Lungenfunktionsdiagnostik zur Feststellung einer Obstruktion der Atemwege bei Kindern und Jugendlichen [Significance of contemporary methods of lung function testing for the detection of airway obstruction in children and adolescents]. Z Erkr Atmungsorgane 1977; 149: 343-371. 
36 Modi AC, Lim CS, Yu N, et al. A multi-method assessment of treatment adherence for children with cystic fibrosis. J Cyst Fibros 2006; 5: 177-185.

37 Burrows JA, Bunting JP, Masel PJ, et al. Nebulised dornase alpha: adherence in adults with cystic fibrosis. J Cyst Fibros 2002; 1 : 255-259.

38 Latchford G, Duff A, Quinn J, et al. Adherence to nebulised antibiotics in cystic fibrosis. Patient Educ Couns 2009; 75: 141-144.

39 Ramsey BW, Astley SJ, Aitken ML, et al. Efficacy and safety of short-term administration of aerosolized recombinant human deoxyribonuclease in patients with cystic fibrosis. Am Rev Respir Dis 1993; 148: 145-151.

40 Shah PL, Scott SF, Geddes DM, et al. An evaluation of two aerosol delivery systems for rhDNase. Eur Respir J 1997; 10: 1261-1266.

41 Geller DE, Eigen H, Fiel SB, et al. Effect of smaller droplet size of dornase alfa on lung function in mild cystic fibrosis. Dornase Alfa Nebulizer Group. Pediatr Pulmonol 1998; 25: 83-87.

42 Geller D, Thipphawong J, Otulana B, et al. Bolus inhalation of rhDNase with the AERx system in subjects with cystic fibrosis. J Aerosol Med 2003; 16: 175-182. 